As the Nation's principal conservation agency, the Department of the Interior has responsibility for most of our nationally owned public lands and natural resources. This includes fostering the wisest use of our land and water resources, protecting our fish and wildlife, preserving the environmental and cultural values of our national parks and historical places, and providing for the enjoyment of life through outdoor recreation. The Department assesses our energy and mineral resources and works to assure that their development is in the best interests of all our people. The Department also has a major responsibility for American Indian reservation communities and for people who live in Island Territories under U.S. administration.
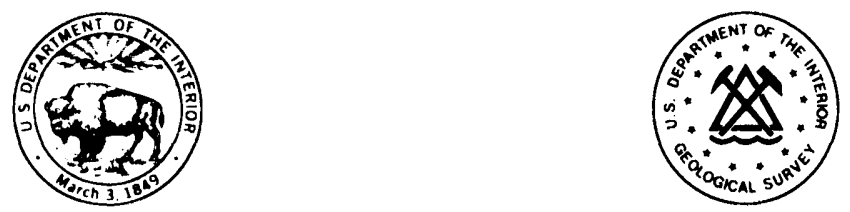

\section{Elevations and Distances \\ in the United States}




\section{Elevations \\ and Distances \\ in the United States}

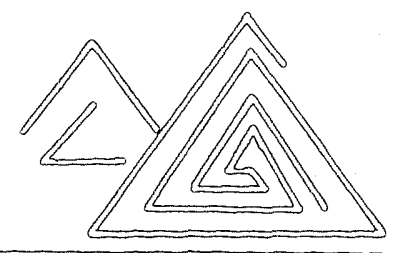

The information in this booklet was compiled to answer inquiries received by the Geological Survey from students; teachers; writers; editors; pub- lishers of encyclopedias, almanacs and other reference books; and people in many other fields of work.

The elevations of features and distances between points in the United States were determined from surveys and topographic maps of the Geological Survey or obtained from other sources. Some of this information, compiled from older maps, will be revised as new surveys are completed. For further information, write to the U. S. Geological Survey, 507 National Center, Reston,Virginia 22092

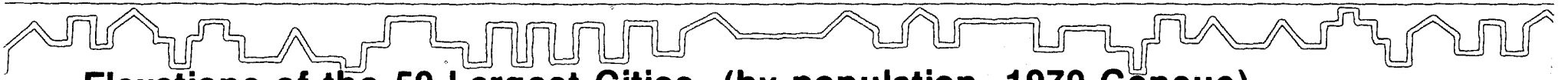

\section{Elevations of the 50 Largest Cities (by population, 1970 Census)}

\begin{tabular}{llrrr}
\multicolumn{1}{c}{ City } & \multicolumn{1}{c}{ State } & Rank & $\begin{array}{r}\text { High } \\
\text { Feet }\end{array}$ & $\begin{array}{r}\text { Low } \\
\text { Feet }\end{array}$ \\
New York & New York & 1 & 410 & Sea level \\
Chicago & Illinois & 2 & 673 & 1577 \\
Los Angeles & California & 3 & 5,074 & Sea level \\
Philadelphia & Pennsylvania & 4 & 441 & Sea level \\
Detroit & Michigan & 5 & 672 & 1573 \\
Houston & Texas & 6 & 83 & Sea level \\
Baltimore & Maryland & 7 & 489 & Sea level \\
Dallas & Texas & 8 & 686 & 390 \\
Washington & D. C. & 9 & 410 & 1 \\
Cleveland & Ohio & 10 & 1,050 & 1570 \\
Indianapolis & Indiana & 11 & 845 & 664 \\
Milwaukee & Wisconsin & 12 & 799 & 1578 \\
San Francisco & California & 13 & 934 & Sea level \\
San Diego & California & 14 & 823 & Sea level \\
San Antonio & Texas & 15 & 1,000 & 505 \\
Boston & Massachusetts & 16 & 330 & Sea level \\
Memphis & Tennessee & 17 & 335 & 195 \\
St. Louis & Missouri & 18 & 614 & 385 \\
New Orleans & Louisiana & 19 & 25 & -5 \\
Phoenix & Arizona & 20 & 1,160 & 1,058 \\
Columbus & Ohio & 21 & 893 & 685 \\
Seattle & Washington & 22 & 520 & Sea level \\
Jacksonville & Florida & 23 & 40 & Sea level \\
Pittsburgh & Pennsylvania & 24 & 1,370 & 710 \\
Denver & Colorado & 25 & 5,470 & 5,130 \\
Kansas City & Missouri & 26 & 1,020 & 720 \\
& & & &
\end{tabular}

City

Atlanta

Buffalo

Cincinnati

Nashville-Davidson

San Jose

Minneapolis

Ft. Worth

Toledo

Newark

Portland

Oklahoma City

Louisville

Oakland

Long Beach

Omaha

Miami

Tulsa

Honolulu

El Paso

St. Paul

Norfolk

Birmingham

Rochester

Tampa

\footnotetext{
${ }^{1}$ Water level, Great Lakes.
} 


\section{Extreme and Mean Elevations}

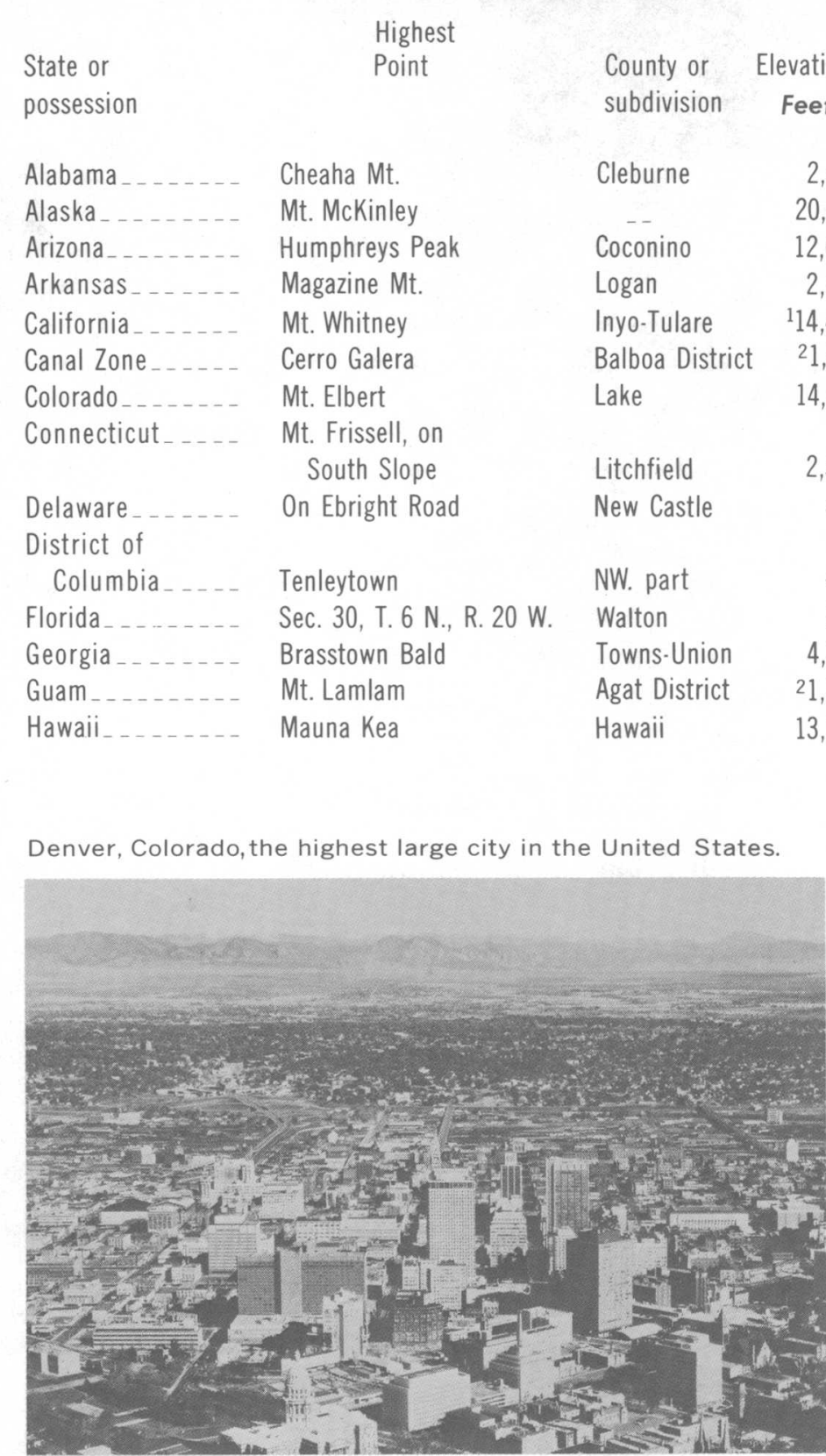

Death Valley, California, lowest point in the United States.

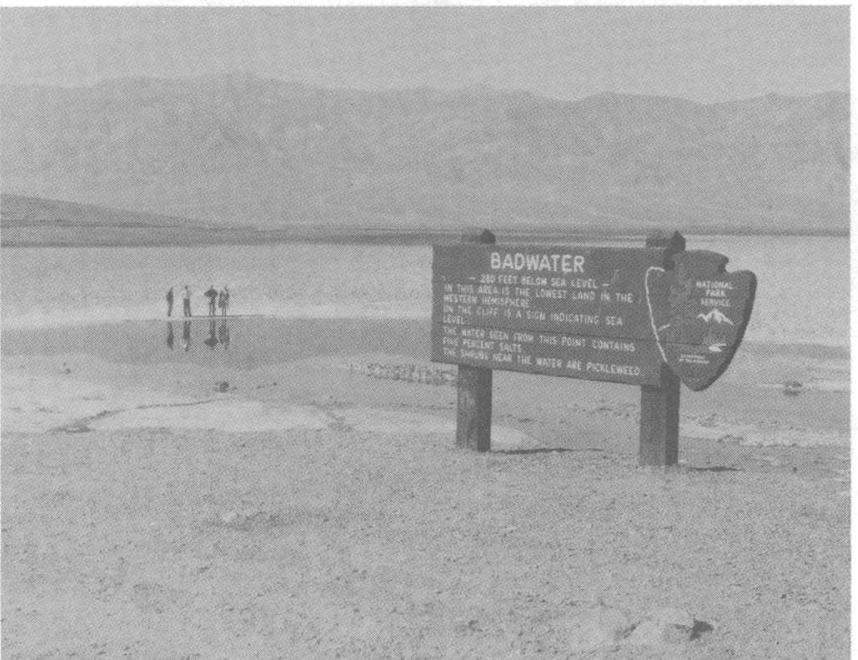


Mount McKinley in Alaska is the loftiest peak in North America.

\begin{tabular}{|c|c|c|c|c|c|c|c|}
\hline $\begin{array}{l}\text { State or } \\
\text { possession }\end{array}$ & $\begin{array}{l}\text { Highest } \\
\text { Point }\end{array}$ & $\begin{array}{l}\text { County or } \\
\text { subdivision }\end{array}$ & $\begin{array}{c}\text { Elevation } \\
\text { Feet }\end{array}$ & $\begin{array}{l}\text { Lowest } \\
\text { Point }\end{array}$ & $\begin{array}{l}\text { County or } \\
\text { subdivision }\end{array}$ & $\begin{array}{c}\text { Elevation } \\
\text { Feet }\end{array}$ & $\begin{array}{c}\text { mean } \\
\text { elevation } \\
\text { Feet }\end{array}$ \\
\hline Idaho _......... & Borah Peak & Custer & 12,662 & Snake River & Nez Perce & 710 & 5,000 \\
\hline Illinois_. _. _. _. & Charles Mound & Jo Daviess & 1,235 & Mississippi River & Alexander & 2279 & 600 \\
\hline Indiana _. . . . . . & Franklin Township & Wayne & 1,257 & Ohio River & Posey & 2320 & 700 \\
\hline lowa $\ldots . . . . . . .$. & Sec. 29, T. 100 N., R. 41 W. & Osceola & 1,670 & Mississippi River & Lee & 480 & 1,100 \\
\hline Kansas_......... & Mt. Sunflower & Wallace & 34,039 & Verdigris River & Montgomery & 680 & 2,000 \\
\hline Kentucky _...... & Black Mt. & Harlan & 4,145 & Mississippi River & Fulton & 2257 & 750 \\
\hline Louisiana_....... & Driskill Mt. & Bienville & 535 & New Orleans & Orleans & -5 & 100 \\
\hline Maine _........ & Mt. Katahdin & Piscataquis & 5,268 & Atlantic Ocean & -- & Sea level & 600 \\
\hline Maryland . . . . . . & Backbone Mt. & Garrett & 3,360 & Atlantic Ocean & -- & Sea level & 350 \\
\hline Massachusetts_. _. & Mt. Greylock & Berkshire & 3,491 & Atlantic Ocean & -- & Sea level & 500 \\
\hline Michigan _....... & Mt. Curwood & Baraga & 1,980 & Lake Erie & -- & 2572 & 900 \\
\hline Minnesota _...... & Eagle Mt. & Cook & 2,301 & Lake Superior & -- & 602 & 1,200 \\
\hline Mississippi _...... & Woodall Mt. & Tishomingo & 806 & Gulf of Mexico & -- & Sea level & 300 \\
\hline Missouri _. _. _. _. & Taum Sauk Mt. & Iron & 1,772 & St. Francis River & Dunklin & 2230 & 800 \\
\hline Montana & Granite Peak & Park & 12,799 & Kootenai River & Lincoln & 1,800 & 3,400 \\
\hline Nebraska $\ldots \ldots$ & Johnson Township & Kimball & 5,426 & SE corner of State & Richardson & 840 & 2,600 \\
\hline Nevada ......... & Boundary Peak & Esmeralda & 13,143 & Colorado River & Clark & 470 & 5,500 \\
\hline New Hampshire _. - & Mt. Washington & Coos & 6,288 & Atlantic Ocean & -- & Sea level & 1,000 \\
\hline New Jersey _..... & High Point & Sussex & ${ }^{3} 1,803$ & Atlantic Ocean & -- & Sea level & 250 \\
\hline New Mexico _.... & Wheeler Peak & Taos & 13,161 & Red Bluff Res. & Eddy & 2,817 & 5,700 \\
\hline New York_...... & Mt. Marcy & Essex & 5,344 & Atlantic Ocean & -- & Sea level & 1,000 \\
\hline North Carolina . - & Mt. Mitchell & Yancey & 6,684 & Atlantic Ocean & -- & Sea level & 700 \\
\hline North Dakota & White Butte & Slope & 3,506 & Red River & Pembina & 750 & 1,900 \\
\hline Ohio _.......... & Campbell Hill & Logan & 1,550 & Ohio River & Hamilton & 2433 & 850 \\
\hline Oklahoma & Black Mesa & Cimarron & 4,973 & Little River & McCurtain & 287 & 1,300 \\
\hline Oregan & Mt. Hood & $\begin{array}{l}\text { Clackamas- } \\
\text { Hood River }\end{array}$ & 11,239 & Pacific Ocean & -- & Sea level & 3,300 \\
\hline Pennsylvania _._._ & Mt. Davis & Somerset & 3,213 & Delaware River & Delaware & Sea level & 1,100 \\
\hline Puerto Rico. . . _- & Cerro de Punta & Ponce District & 4,389 & Atlantic Ocean & - - & Sea level & 1,800 \\
\hline Rhode Island _... & Jerimoth Hill & Providence & 812 & Atlantic Ocean & -- & Sea level & 200 \\
\hline Samoa & Lata Mt. & Tau Island & 3,160 & Pacific 0cean & -- & Sea level & 1,300 \\
\hline South Carolina & Sassafras Mt. & Pickens & 3,560 & Atlantic Ocean & -. & Sea level & 350 \\
\hline
\end{tabular}




\begin{tabular}{|c|c|c|c|c|c|c|c|}
\hline $\begin{array}{l}\text { State or } \\
\text { possession }\end{array}$ & $\begin{array}{l}\text { Highest } \\
\text { Point }\end{array}$ & $\begin{array}{l}\text { County or } \\
\text { subdivision }\end{array}$ & $\begin{array}{c}\text { Elevation } \\
\text { Feet }\end{array}$ & $\begin{array}{l}\text { Lowest } \\
\text { Point }\end{array}$ & $\begin{array}{l}\text { County or } \\
\text { subdivision }\end{array}$ & $\begin{array}{c}\text { Elevation } \\
\text { Feet }\end{array}$ & $\begin{array}{c}\text { mean } \\
\text { elevation } \\
\text { Feet }\end{array}$ \\
\hline South Dakota _..._- & Harney Peak & Pennington & 7,242 & Big Stone Lake & Roberts & 962 & 2,200 \\
\hline Tennessee . . . . . & Clingmans Dome & Sevier & 6,643 & Mississippi River & Shelby & 2182 & 900 \\
\hline Texas_......... & Guadalupe Peak & Culberson & 8,749 & Gulf of Mexico & -- & Sea level & 1,700 \\
\hline Utah . . . . . . . & Kings Peak & Duchesne & 13,528 & Beaverdam Creek & Washington & 2,000 & 6,100 \\
\hline Vermont......... & Mt. Mansfield & Lamoille & 4,393 & Lake Champlain & Franklin & 95 & 1,000 \\
\hline Virginia _. . . . . . & Mt. Rogers & Grayson-Smyth & 5,729 & Atlantic Ocean & - & Sea level & 950 \\
\hline Virgin Islands _... & Crown Mt. & St. Thomas Island & 1,556 & Atlantic Ocean & -- & Sea level & 750 \\
\hline Washington...... & Mt. Rainier & Pierce & 14,410 & Pacific Ocean & -. & Sea level & 1,700 \\
\hline West Virginia _... & Spruce Knob & Pendleton & 4,863 & Potomac River & Jefferson & 240 & 1,500 \\
\hline Wisconsin ....... & Timms Hill & Price & 1,951 & Lake Michigan & _- & 2581 & 1,050 \\
\hline Wyoming _...... & Gannett Peak & Fremont & 13,804 & Belle Fourche River & Crook & 3,100 & 6,700 \\
\hline United States_... & Mt. McKinley, Alaska & -- & 20,320 & Death Valley & Inyo, Calif. & -282 & 2,500 \\
\hline${ }^{1}$ National Geodeti & Survey. $\quad 20$ & of Engineers. & & 3 State Surveys. & & & \\
\hline
\end{tabular}

Elevations of Named Summits over 14,000 feet above sea level

\begin{tabular}{|c|c|c|c|c|c|c|c|}
\hline Summit & State & Quadrangle & $\begin{array}{c}\text { Elevation } \\
\text { Feet }\end{array}$ & Summit & State & Quadrangle & $\begin{array}{c}\text { Elevation } \\
\text { Feet }\end{array}$ \\
\hline Mt. McKinley & Alaska & Mt. McKinley A-3 & 20,320 & Blanca Peak & Colo. & Blanca Peak & 214,345 \\
\hline Mt. St. Elias & Alaska & Mt. St. Elias & 18,008 & La Plata Peak & Colo. & Mt. Elbert & 14,336 \\
\hline Mt. Foraker & Alaska & Talkeetna D-3 & 17,400 & Uncompahgre Peak & Colo. & Lake City & 14,309 \\
\hline Mt. Bona & Alaska & McCarthy B-2 & 16,421 & Crestone Peak & Colo. & Crestone Peak & 14,294 \\
\hline Mt. Blackburn & Alaska & McCarthy $\mathrm{C}-7$ & 16,390 & Mt. Lincoln & Colo. & Alma & 14,286 \\
\hline Mt. Sanford & Alaska & Gulkana A-1 & 16,237 & Grays Peak & Colo. & Grays Peak & 14,270 \\
\hline South Buttress & Alaska & Mt. McKinley A-2 & 15,885 & Mt. Antero & Colo. & Poncha Springs & 14,269 \\
\hline Mt. Vancouver & Alaska & Mt. St. Elias & 15,700 & Torreys Peak & Colo. & Grays Peak & 14,267 \\
\hline Mt. Churchill & Alaska & McCarthy B-2 & 15,638 & Castle Peak & Colo. & Hayden Peak & 14,265 \\
\hline Mt. Fairweather & Alaska & Mt. Fairweather & 15,300 & Quandary Peak & Colo. & Breckenridge & 14,265 \\
\hline Mt. Hubbard & Alaska & Mt. St. Elias & 115,015 & Mt. Evans & Colo. & Mt. Evans & 14,264 \\
\hline Mt. Bear & Alaska & McCarthy B-1 & 14,831 & Longs Peak & Colo. & Longs Peak & 14,255 \\
\hline East Buttress & Alaska & Mt. McKinley A-2 & 14,730 & Mt. Wilson & Colo. & Mt. Wilson & 14,246 \\
\hline Mt. Hunter & Alaska & Talkeetna D-3 & 14,573 & White Mt. & Calif. & White Mt. & 114,246 \\
\hline Mt. Alverstone & Alaska & Mt. St. Elias & 114,565 & North Palisade & Calif. & Mt. Goddard & 14,242 \\
\hline Browne Tower & Alaska & Mt. McKinley A-2 & 14,530 & Shavano Peak & Colo. & Poncha Springs & 14,229 \\
\hline Mt. Whitney & Calif. & Mt. Whitney & 214,494 & Mt. Belford & Colo. & Mt. Harvard & 14,197 \\
\hline Mt. Elbert & Colo. & Mt. Elbert & 214,433 & Mt. Princeton & Colo. & Poncha Springs & 14,197 \\
\hline Mt. Massive & Colo. & Mt. Massive & 14,421 & Crestone Needle & Colo. & Crestone Peak & 14,197 \\
\hline Mt. Harvard & Colo. & Mt. Harvard & 14,420 & Mt. Yale & Colo. & Mt. Harvard & 14,196 \\
\hline Mt. Rainier & Wash. & Mt. Rainier & 14,410 & Mt. Bross & Colo. & Alma & 14,172 \\
\hline Mt. Williamson & Calif. & Mt. Whitney & 14,375 & Kit Carson Mt. & Colo. & Crestone Peak & 14,165 \\
\hline
\end{tabular}




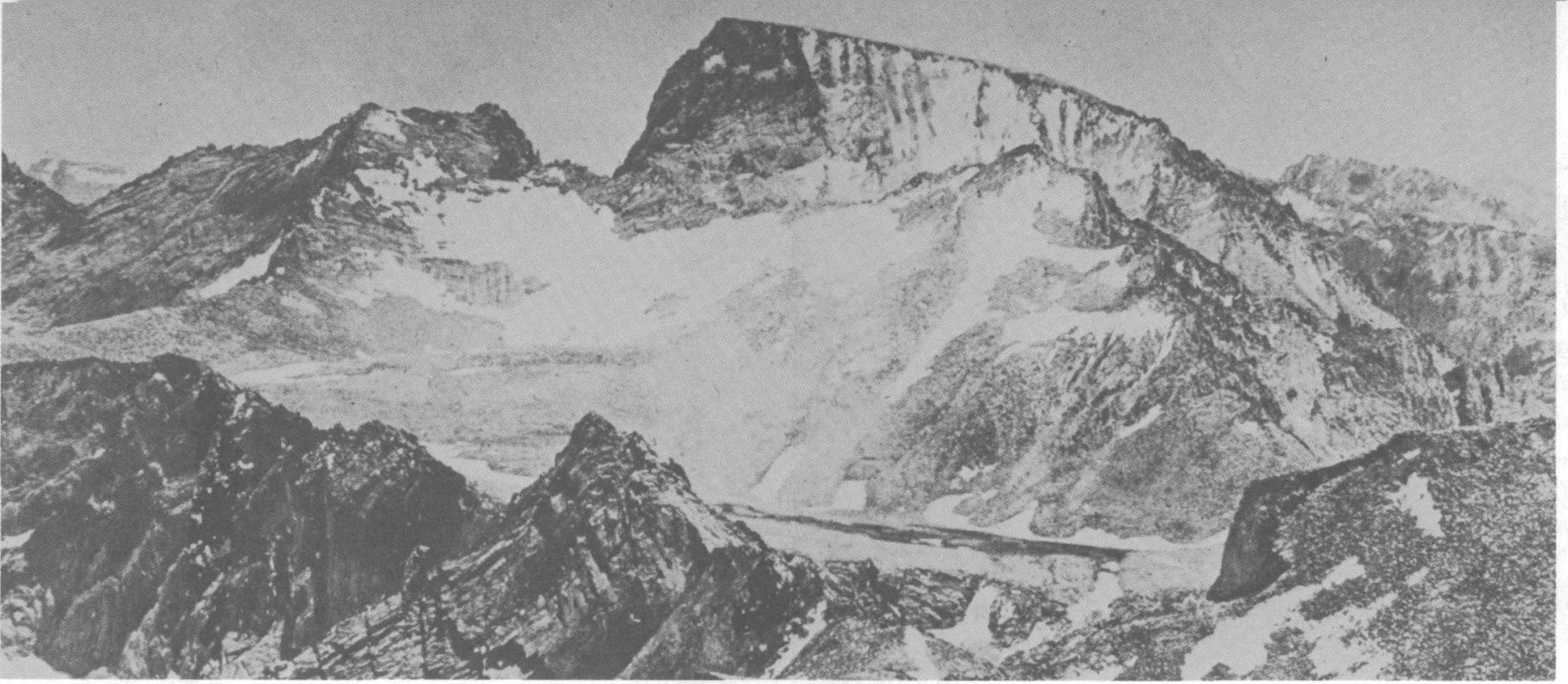

Mount Whitney, highest point in the conterminous 48 States, is less than 80 miles from Death Valley, which is the lowest point.

\begin{tabular}{llll}
\multicolumn{1}{c}{ Summit } & State & \multicolumn{1}{c}{ Quadrangle } & $\begin{array}{c}\text { Elevation } \\
\text { Feet }\end{array}$ \\
Mt. Wrangell & Alaska & Gulkana A-1 & 14,163 \\
Mt. Shasta & Calif. & Shasta & 14,162 \\
Mt. Sill & Calif. & Mt. Goddard & 14,162 \\
El Diente Peak & Colo. & Dolores Peak & 14,159 \\
Maroon Peak & Colo. & Maroon Bells & 14,156 \\
Tabeguache Mt. & Colo. & Garfield-Poncha \\
& \multicolumn{3}{c}{ Springs } \\
Mt. Oxford & Colo. & Mt. Harvard & 14,155 \\
Mt. Sneffels & Colo. & Mt. Sneffels & 14,153 \\
Mt. Democrat & Colo. & Climax \\
Capitol Peak & Colo. & Capitol Peak & 14,150 \\
Pikes Peak & Colo. & Pikes Peak & 14,148 \\
Snowmass Mt. & Colo. & Snowmass Mt. & 14,130 \\
Windom Peak & Colo. & Needle Mts. & 14,110 \\
Mt. Russell & Calif. & Mt. Whitney & 14,092 \\
Mt. Eolus & Colo. & Needle Mts. & 14,086 \\
Mt. Columbia & Colo. & Mt. Harvard & 14,084 \\
Mt. Augusta & Alaska & Mt. St. Elias & 14,073 \\
Missouri Mt. & Colo. & Mt. Harvard & 14,070 \\
Humboldt Peak & Colo. & Crestone Peak & 14,067 \\
Mt. Bierstadt & Colo. & Mt. Evans & 14,064 \\
& & & 14,060
\end{tabular}

1Elevation determined in 1966. Not shown on Mt. St.Elias Quadrangle. 


\section{Elevations of Selected Summits East of the Rocky Mountains}

Summit

State

White Mountains, Presidential Range:

Mt. Washington

Mt. Adams

Mt. Jefferson

Mt. Sam Adams

Mt. Clay

Boott Spur

Mt. Monroe

Mt. Madison

Mt. Lafayette

Mt. Lincoln

Mt. Franklin

South Twin Mt.

Mt. Quincy Adams

$\begin{array}{ll}\text { New Hampshire } & \text { Coos } \\ \text { New Hampshire } & \text { Coos } \\ \text { New Hampshire } & \text { Coos } \\ \text { New Hampshire } & \text { Coos } \\ \text { New Hampshire } & \text { Coos } \\ \text { New Hampshire } & \text { Coos } \\ \text { New Hampshire } & \text { Coos } \\ \text { New Hampshire } & \text { Coos } \\ \text { New Hampshire } & \text { Grafton } \\ \text { New Hampshire } & \text { Grafton } \\ \text { New Hampshire } & \text { Coos } \\ \text { New Hampshire } & \text { Grafton } \\ \text { New Hampshire } & \text { Coos }\end{array}$

Adirondack Mountains, Mt. Marcy area:

Mt. Marcy

Algonquin Peak

Mt. Haystack

Mt. Skylight

Whiteface Mt

Dix Mt.

Boundary Peak

Gray Peak

Iroquois Peak

Basin Mt.

Little Marcy

Gothics

Catskill Mountains:

Slide Mt.

Hunter Mt.

Black Dome

Blackhead

Thomas Cole Mt.

West Kill Mt.

Graham Mt.

Cornell Mt.
New York

New York

New York

New York

New York

New York

New York

New York

New York

New York

New York

New York

New York

New York

New York

New York

New York

New York

New York

New York
County

Quadrangle

Mt. Washington

Mt. Washington

Mt. Washington

Mt. Washington

Mt. Washington

Mt. Washington

Mt. Washington

Mt. Washington

Franconia

Franconia

Crawford Notch

Franconia

Mt. Washington

Essex

Essex

Essẹx

Essex

Essex

Essex

Essex

Essex

Essex

Essex

Essex

Essex

Ulster

Greene

Greene

Greene

Greene

Greene

Ulster

Ulster
Mt. Marcy

Mt. Marcy

Mt. Marcy

Mt. Marcy

Lake Placid

Mt. Marcy

Mt. Marcy

Mt. Marcy

Mt. Marcy

Mt. Marcy

Mt. Marcy

Mt. Marcy

Peekamoose

Hunter

Freehold

Freehold

Hensonville

Lexington

Seager

Phoenicia
Scale

$1: 62,500$

$1: 62,500$

1:62,500

1:62,500

1:62,500

1:62,500

1:62,500

$1: 62,500$

$1: 62,500$

$1: 62,500$

$1: 62,500$

$1: 62,500$

1:62,500

1:62,500

$1: 62,500$

$1: 62,500$

$1: 62,500$

$1: 62,500$

$1: 62,500$

$1: 62,500$

$1: 62,500$

$1: 62,500$

$1: 62,500$

$1: 62,500$

1:62,500

$1: 24,000$

$1: 24,000$

$1: 24,000$

$1: 24,000$

1:24,000

$1: 24,000$

$1: 24,000$

$1: 24,000$
Elevation

Feet

6,288

5,798

5,715

5,580

5,532

5,500

5,385

5,363

5,249

5,108

5,004

4,926

4,920

5,344

5,114

4,960

4,926

4,867

4,857

4,840

4,840

4,840

4,827

4,765

4,736

4,180

4,040

3,980

3,940

3,940

3,880

3,868

3,860 
Doubletop Mt.

New York

Table Mt.

New York

Peekamoose Mt.

New York

Plateau Mt.

County
Ulster
Ulster
Ulster
Greene

Blue Ridge and Great Smoky Mountains:

Mt. Mitchell
Mt. Craig
Clingmans Dom
Mt. Guyot
Balsam Cone
Cattail Peak
Mt. Le Conte
Big Tom
Mt. Gibbes
Potato Hill
Mt. Chapman

Old Black

\section{Celo Knob}

Ozark-Ouachita Mountains:

Magazine Mt.

Rich Mt.

Poteau Mt.

Black Fork Mt.

Sugar Loaf Mt.

Buffalo Lookout

Wilton Mt.

Turner Ward Knob

Kiamichi Mt:

Winding Stair Mt.

Lynn Mt.

Reeves Mt.

Black Hills:

Harney Peak ${ }^{2}$

Bear Mt.

Crooks Tower

Terry Peak

Crows Nest Peak

\section{North Carolina \\ North Carolina \\ North Carolina- \\ Tennessee}

Tennessee

North Carolina

North Carolina

Tennessee

North Carolina

North Carolina

North Carolina

North Carolina-

Tennessee

North Carolina-

Tennessee

North Carolina

\section{Arkansas \\ Oklahoma \\ Arkansas \\ Arkansas \\ Oklahoma \\ Arkansas \\ Oklahoma \\ Arkansas \\ Oklahoma \\ Oklahoma \\ Oklahoma \\ Arkansas}

South Dakota

South Dakota

South Dakota

South Dakota

South Dakota
North Carolina-
Yancey
Yancey
Swain
Sevier

Haywood

Sevier

Yancey

Yancey

Sevier

Yancey

Yancey

Yancey

Swain

Sevier

Haywood

Cocke-Sevier

Yancey

Logan

Logan

Sebastian

Polk

Le Flore

Newton

Le Flore

Newton

Le Flore

Le Flore

Le Flore

Newton

Pennington

Pennington

Lawrence

Lawrence

Pennington
Seager

Peekamoose

Peekamoose

Hunter

Mt. Mitchell

Mt. Mitchell

Clingmans

Dome

Mt. Guyot

Mt. Mitchell

Mt. Mitchell

Mt. Le Conte

Mt. Mitchell

Montreat

Mt. Mitchell

Mt. Guyot

Mt. Guyot

Celo

Blue Mt.

Potter

Cauthron

Potter

Hartford

Fallsville

Page

Fallsville

Muse

Page

Page

Boxley

Custer

Berne

Crooks Tower

Lead

Crows Nest Peak
$1: 24,000$

1:24,000

$1: 24,000$

1:24,000

1:24,000

$1: 24,000$

$1: 24,000$

1:24,000

$1: 24,000$

$1: 24,000$

$1: 24,000$

$1: 24,000$

$1: 24,000$

$1: 24,000$

1:24,000

$1: 24,000$

$1: 24,000$

1:24,000

$1: 62,500$

$1: 24,000$

$1: 62,500$

1:24,000

$1: 24,000$

$1: 62,500$

$1: 24,000$

$1: 24,000$

$1: 62,500$

$1: 62,500$

$1: 24,000$

$1: 24,000$

$1: 24,000$

$1: 24,000$

$1: 24,000$

$1: 24,000$
Elevation Feet

3,860

3,847

3,843

3,840

6,684

6,647

6,643

6,621

6,600

6,600

6,593

6,560

6,520

6,440

6,417

6,370

6,327

2,753

2,681

2,669

2,586

2,564

2,561

2,557

2,463

2,451

2,451

2,440

2,440

7,242

7,166

7,137

7,064

7,048 
White Tail Peak

Flag Mt.

Medicine Mt.

Nipple Butte

Custer Peak

Mt. Rushmore

Devils Tower

${ }^{1}$ Highest summit east of the Mississippi River.
Pennington

Pennington

Pennington

Pennington

Lawrence

Pennington

Crook
Deerfield

$1: 24,000$

$1: 24,000$

$1: 24,000$

$1: 24,000$

$1: 24,000$

$1: 24,000$

Devils Tower $\quad 1: 62,500 \quad 5,112$

Mt. Rushmore $\quad 1: 24,000 \quad 5,725$

${ }^{2}$ Highest summit east of the Rocky Mountains.

\section{Geographic Centers}

Because there is no generally accepted definition of a geographic center and no completely satisfactory method for determining it, there may be as many geographic centers of a State or country as there are definitions of the term.

The geographic center of an area may be defined as the center of gravity of the surface, or that point on which the surface of the area would balance if it were a plane of uniform thickness. Logan Boone Indiana Story lowa Kansas Barton Kentucky Marion
State County Chilton

Alabama

Alaska

$63^{\circ} 50^{\prime} \mathrm{N}$., $152^{\circ} 00^{\prime} \mathrm{W}$., 60 miles northwest of Mt. McKinley Arizona Yavapai

Arkansas Pulaski 55 miles east-southeast of Prescott

California Madera 12 miles northwest of Little Rock Colorado Park 38 miles east of Madera Connecticut. Hartford 30 miles northwest of Pikes Peak Delaware Kent At East Berlin

District of Columbia

Florida

Georgia Hernando

Twiggs Hawaii Custer

Locality

12 miles southwest of Clanton

Hawail

Idaho
11 miles south of Dover Near Fourth and L Streets NW 12 miles north-northwest of Brooksville 18 miles southeast of Macon $20^{\circ} 15^{\prime} \mathrm{N} ., 156^{\circ} 20^{\prime} \mathrm{W}$., off Maui Island At Custer, southwest of Challis 28 miles northeast of Springfield 14 miles north-northwest of Indianapolis 5 miles northeast of Ames 15 miles northeast of Great Bend 3 miles north-northwest of Lebanon

Curvature of the Earth, large bodies of water, irregular surfaces, and other factors affect the determination of center of gravity.

In determining the centers of the States, islands adjacent to their coastlines and large bodies of water on their boundaries were excluded.

The geographic centers and positions listed below should be considered as approximations.

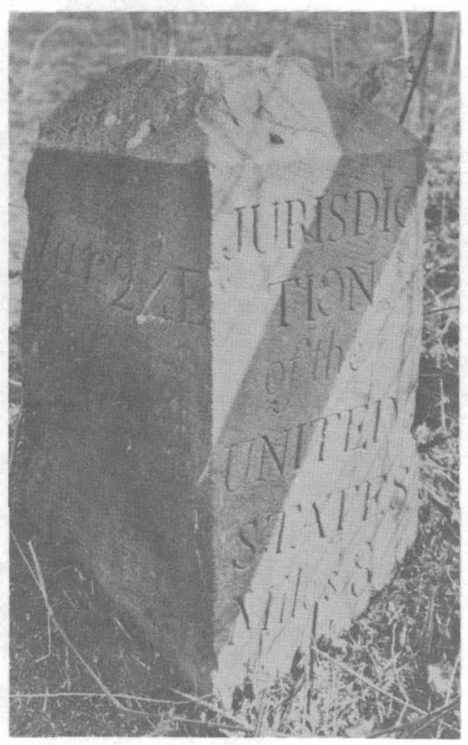

Original Federal boundary marker of the District of Columbia. 
Louisiana

Maine

Maryland

Massachusetts

Michigan

Minnesota

Mississippi

Missouri.

Montana

Nebraska

Nevada.

New Hampshire

New Jersey.

New Mexico

New York

North Carolina

North Dakota

Ohio

Oklahoma

Oregon

Pennsylvania

Rhode Island

South Carolina

South Dakota

Tennessee

Texas

Utah

Vermont

Virginia

Washington

West Virginia

Wisconsin.

Wyoming
Avoyelles

Piscataquis

Prince Georges

Worcester

Wexford

Crow Wing

Leake

Miller

Fergus

Custer

Lander

Belknap

Mercer

Torrance

Madison

Chatham

Sheridan

Delaware

Oklahoma

Crook

Centre

Kent.

Richland

Hughes

Rutherford

McCulloch

Sanpete

Washington

Buckingham

Chelan

Braxton

Wood

Fremont.
3 miles southeast of Marksville 18 miles north of Dover

$4 \frac{1}{2}$ miles northwest of Davidsonville

North part of City of Worcester 5 miles north-northwest of Cadillac 10 miles southwest of Brainerd 9 miles west-northwest of Carthage 20 miles southwest of Jefferson City 12 miles west of Lewistown 10 miles northwest of Broken Bow _26 miles southeast of Austin 3 miles east of Ashland 5 miles southeast of Trenton 12 miles south-southwest of Willard $-12+$ miles south of Oneida and $26+$ miles southwest of Utica 10 miles northwest of Sanford 5 miles southwest of McClusky 25 miles north-northeast of Columbus -8 miles north of Oklahoma City 25 miles south-southeast of Prineville $2 \frac{1}{2}$ miles southwest of Bellefonte 1 mile south-southwest of Crompton 13 miles southeast of Columbia 8 miles northeast of Pierre .5 miles northeast of Murfreesboro 15 miles northeast of Brady 3 miles north of Manti 3 miles east of Roxbury 5 miles southwest of Buckingham 10 miles west-southwest of Wenatchee -4 miles east of Sutton 9 miles southeast of Marshfield 58 miles east-northeast of Lander
Latitude Longitude

Conterminous United States (48 States) Near Lebanon, Smith County, Kansas $103^{\circ} 38^{\prime}$

Continental United States (49 States) $103^{\circ} 46^{\prime}$

The United States (50 States) $39^{\circ} 50^{\prime}$ $44^{\circ} 59^{\prime}$ Near Castle Rock, Butte County, South Dakota $44^{\circ} 58^{\prime}$ West of Castle Rock, Butte County, South Dakota $48^{\circ} 10^{\prime}$

$98^{\circ} 35^{\prime}$

6 miles west of Balta, Pierce County, North Dakota 
No marked or monumented point has been established by any government agency as the geographic center of either the 50 States, the conterminous United States, or the North American Continent. However, a monument was erected in Lebanon, Kansas, by a group of citizens who hired engineers to determine the position of the "geographic center" of the United States.

Sometimes confused with the geographic center of the United States is the reference point for all property lines and city, county, State, and international boundaries on the North American Continent that are tied to the National Triangulation networks of the United States, Canada, Mexico, and Central America. This point is Meades Ranch Triangulation station located at latitude $39^{\circ} 13^{\prime} 26.686^{\prime \prime}$, longitude $98^{\circ} 32^{\prime} 30.506^{\prime \prime}$, about 12 miles north of Lucas, Kansas. It is the base point or origin of geodetic positions and directions in the triangulation net of the United States because it is at the junction of the main east-west transcontinental triangulation arc, which stretches from the Atlantic to the Pacific Coasts, and the main north-south arc, which follows approximately the 98th meridian from the Canadian border to the Rio Grande.

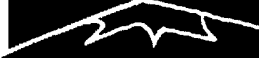 \\ Distances from Extreme Polnts to Geographic Centers \\ r}

Extreme point

\begin{tabular}{lc}
\multicolumn{2}{c}{ Coordinates } \\
Latitude & Longitude \\
(N) & (W)
\end{tabular}

$71^{\circ} 23^{\prime}-156^{\circ} 29^{\prime}$

$51^{\circ} 19^{\prime}$

$179^{\circ} 55^{\prime}(\mathrm{E})$

$51^{\circ} 17^{\prime} \quad 179^{\circ} 09^{\prime}$

$52^{\circ} 55^{\prime}$

$172^{\circ} 27^{\prime}(\mathrm{E})$

$49^{\circ} 23^{\prime}$

$95^{\circ} 09^{\prime}$

$47^{\circ} 04^{\prime}$

$44^{\circ} 49^{\prime}$

$24^{\circ} 33^{\prime}$

$.25^{\circ} 07^{\prime}$

$25^{\circ} 29^{\prime}$
Direction
from geographic centers
Geographic

50 States West of Castle Rock Butte County, South Dakota $44^{\circ} 58^{\prime} \mathrm{N}-103^{\circ} 46^{\prime}$ Statute miles

Northwest

2,502

Cape Sable, Florida.......

Log Point, Elliot Key,

Florida 
Latitude Longitude

(N)
East point, St. Croix, Virgin Islands

Extreme south point of Texas

Steps Point, American Samoa

Orote Point, Guam . . . . . . .

Kure Island, Hawaii

Ka Lae, Hawaii.

Cape Mendocino, Calif.

Cape Alava, Washington $17^{\circ} 45^{\prime}$

$25^{\circ} 50^{\prime}$

$14^{\circ} 23^{\prime}(\mathrm{S})$

$13^{\circ} 27^{\prime}$

$28^{\circ} 25^{\prime}$

$18^{\circ} 56^{\prime}$

$40^{\circ} 27^{\prime}$

$48^{\circ} 10^{\prime}$

${ }^{1}$ General direction to Lake of the Woods Projection is north.

Southeast

$97^{\circ} 24^{\prime}$

$170^{\circ} 46^{\prime}$

$144^{\circ} 37^{\prime}(\mathrm{E})$

$178^{\circ} 22^{\prime}$

$155^{\circ} 41^{\prime}$

$124^{\circ} 24^{\prime}$

$124^{\circ} 44^{\prime}$

South

Southwest

Southwest

Southwest

Southwest

West

West
Direction from

geographic centers
Geographic

50 States

West of Castle Rock

Butte County,

South Dakota

$44^{\circ} 58^{\prime} \mathrm{N}-103^{\circ} 46^{\prime} \mathrm{W}-44^{\circ} 59^{\prime} \mathrm{N}-103^{\circ}$

Near Castle Rock,

Butte County,

South Dakota

Centers

Statute miles

Statute miles

2,932
1,354
5,849
6,567
4,136
3,456
1,089
1,016

2,927

1,367

5,855

6,573

4,143

3,463

1,095

1,020
48 States

Near Lebanon,

Smith County,

Kansas

${ }^{\circ} 50^{\prime} \mathrm{N}-98^{\circ} 35^{\prime}$
Statute miles

2,536

979

5,943

6,961

4,479

3,657

1,358

1,412

${ }^{2}$ General direction to West Quoddy Head is northeast.

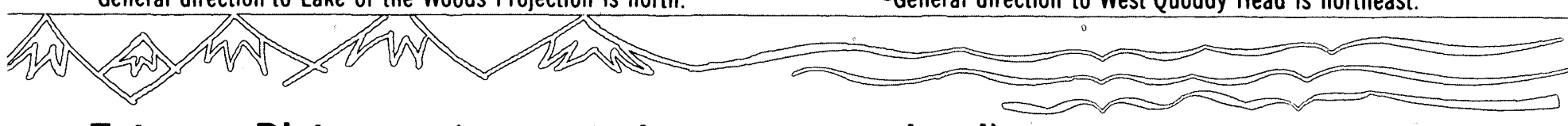

\section{Extreme Distances (computed to mean sea level)}

From

West Quoddy Head, Maine, along the parallel.

West Quoddy Head, Maine

West Quoddy Head, Maine

West Quoddy Head, Maine

Log Point, Elliot Key, Florida

Log Point, Elliot Key, Florida

Mangrove Point, Florida

Mangrove Point, Florida

Rivera Beach, Florida

East Point, St. Croix, Virgin Islands

East Point, St. Croix, Virgin Islands

Approximately 10 miles south of Brunswick, Georgia

th end of Maine-New Brunswick boundary

Point Barrow, Alaska

Southern point of Texas, due north

West point of Amatignak Island, Alaska

\section{Lengths of United States Boundaries}

Stotute miles

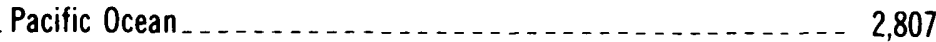

Cape Wrangell, Alaska . . . . . . . . . . . . . . . . . . . . . . 4,839

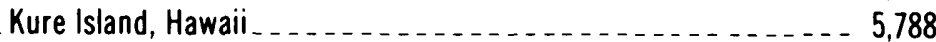

Point Arena, California (farthest points, 48 States) _ _ . . . . . . . 2,897

Kure Island, Hawaii (farthest points, 50 States) _ _ . . _ _ . . . . 5, 5,852

Cape Wrangell, Alaska $\ldots \ldots \ldots \ldots$ 5,495

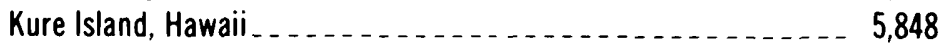

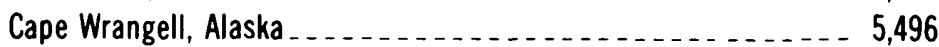

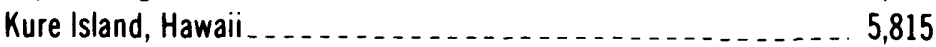

Cape Wrangell, Alaska . . . . . . . . . . . . . . . . . . . . . 5,939

Orote Point, Guam (longest distance including outlying areas) _ . 9,497

Approximately 12 miles south of San Diego, California

(shortest distance 48 States) _. . . . . . . . . . . . . . . . . 2,088

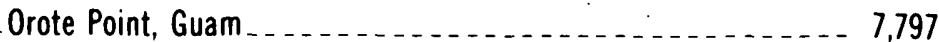

Steps Poinț, Tutuila Island, American Samoa _ . . _ . . _ . _ _ . 5,960

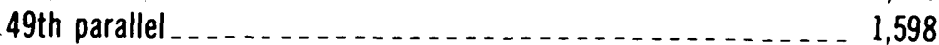

Pochnoi Point, Semisopochnoi Island, Alaska (distance between the most easterly and most westerly points in the United States with reference to the $180^{\circ}$ meridian)

Statute miles

Northern boundary of conterminous United States

Canada - Alaska boundary

Southern boundary of conterminous United States 1,933 